\title{
ERRATUM
}

\section{Temperature impacts on economic growth warrant stringent mitigation policy}

Frances C. Moore and Delavane B. Diaz

Nature Clim. Change 5, 127-131 (2015); published online 12 January 2015; corrected after print 28 January 2015

In the version of this Letter originally published, in equation (1) and in the explanatory sentence following the equation, $j_{\text {TFP }}$ should have $\operatorname{read} r_{\mathrm{TPP}}$. In the second line of the equation, $j_{\mathrm{DJO} j, t}$ should have read $r_{\mathrm{DJO} j, t}$ These errors have been corrected in the online versions of the Letter. 